\title{
Application of Formal Word-Level Analysis to Constrained Random Simulation
}

\author{
Tool Paper
}

\author{
Hyondeuk Kim ${ }^{1,2}$, Hoonsang Jin ${ }^{2}$, Kavita Ravi $^{2}$, Petr Spacek ${ }^{2}$, John Pierce ${ }^{2}$, \\ Bob Kurshan ${ }^{2}$, and Fabio Somenzi ${ }^{1}$ \\ ${ }^{1}$ University of Colorado at Boulder \\ ${ }^{2}$ Cadence Design Systems
}

\begin{abstract}
Constrained random simulation is supported by constraint solvers integrated within simulators. These constraint solvers need to be fast and memory efficient to maintain simulation performance. Binary Decision Diagrams (BDDs) have been successfully applied to represent constraints in this context. However, BDDs are vulnerable to size explosions depending on the constraints they are representing and the number of Boolean variables appearing in them. In this paper, we present a word-level analysis tool DomRed to reduce the number of Boolean variables required to represent constraints by reducing the domain of constraint variables. DomRed employs static analysis techniques to obtain these reductions. We present experimental results to illustrate the impact of this tool.
\end{abstract}

\section{Introduction}

Constrained random simulation is in increasing demand with hardware designers and verification engineers. As the name indicates, it is the simulation of a design under specified constraints. The user is required to capture the behavior of the environment of the design as constraints and the simulation tools simulate the design under these constraints with the aid of constraint solvers embedded in them. Commercial tools, such as Specman, have been popular for providing this capability. To address the need for constrained random simulation, modern hardware description languages (HDL), such as System Verilog, have incorporated constraint specification as part of their syntax.

The overwhelming benefit of constrained random simulation over the traditional writing of testbenches is the automation. Once the constraints are specified, the constraint solver in the simulator enumerates the valid scenarios instead of a human. Further, by specifying weights on the search space, the user can indicate whether the constrained space should be sampled uniformly or specific areas should be focused on.

Given that constraint solving comprises the bulk of constrained random simulation time, the efficiency and performance of constraint solvers is critical. Traditional constraint solving techniques, such as integer linear programming and constraint programming, far lag the performance of simulators. Boolean engines, e.g., BDDs, have been applied quite successfully to this problem[3] by taking advantage of the finite state nature of HDL constraints. More recently, Kitchen and Keuhlmann[2] have provided a 
word-level technique based on Markov-chain Monte Carlo methods. The scalability of this technique to industrial strength designs is yet to be proven.

In our constraint solver, ValueGen, we have incorporated both BDD and SAT-based Boolean engines. BDDs provide the advantage of fast generation of uniformly distributed solutions. However, some constraints have very large BDDs that cause memory explosion during simulation. SAT solvers are less vulnerable to size explosion. On the other hand, each solution generation could be exponentially slower than BDDs.

In this paper, we present a word-level pre-processor, DomRed, that ValueGen applies to the constraints to reduce the size of their representation in the Boolean engines. The pre-processing is a static analysis technique that uses an SMT-like framework. DomRed combines a SAT solver and a linear arithmetic solver that handles primarily integer difference logic, with a minor extension to positive and negative coefficient inequalities. The input to the tool is a Boolean combination of linear arithmetic constraints and bit-vector constraints. The output is a set of variables and their reduced domains. The constraints with reduced-domain variables are then passed on to the Boolean engines, resulting in smaller Boolean representations for constraint solving. We present experimental results of applying DomRed within ValueGen on our simulation testcases.

\section{Constraint Solving in Simulation}

Constraints are Boolean combinations of linear arithmetic and bit-vector expressions on design variables. The expressiveness of the specified constraints is limited by the HDL being used. For example, a System Verilog constraint is

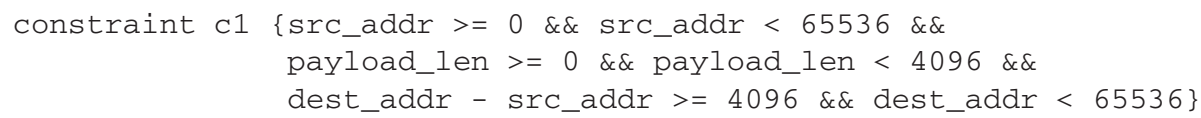

Constraint solving is the task of generating values for the design variables that satisfy the constraints. In the above example, src_addr $=512$, payload_len $=1024$, dest_addr $=4608$ is a set of legal values. Our constraint solver, ValueGen, is invoked dynamically during simulation i.e., every time the simulator encounters a user call to generate new values for variables appearing in constraints, the simulator calls the constraint solver. Tight integration is required between the two to maintain efficiency.

Constraints are typically written on the inputs of the design and may depend on some internal design signals (state variables). During constraint solving, the solver is required to generate values that satisfy both the constraints as well as the states values.

Each set of related HDL constraints, when encountered, is parsed by the simulator, and sent to ValueGen through a word-level API along with the state values. Internally, ValueGen maintains a applies several optimizations at the word-level, including partitioning based on non-overlapping variable support and constant propagation. Finally, it bit-blasts the word-level constraints and calls the Boolean engines (BDD or SAT) on the Boolean representation.

The optimizations in ValueGen result from syntactic and very minor semantic analysis of the constraints. They do not include the ability to deduce that the tightest ranges of 
dest_addr and src_addr in the above example. DomRed addresses exactly this deficiency. It extracts a subset of invariants from semantic analysis of the constraints. If an invariant yields variable bound reductions, then the reduced number of bits are applied to encode the respective variables, the default number of bits are used otherwise.

\section{DomRed: Technical Details}

ValueGen provides DomRed with a quantifier-free first order logic formula with linear arithmetic constraints. An $L A$ constraint is of the form $a_{1} x_{1}+\ldots+a_{n} x_{n} \bowtie c$, where $\bowtie \in\{=, \leq,<,>, \geq, \neq\}$. A difference constraint is a special case of an LA constraint whose form is $x_{i}-x_{j} \bowtie c$. A positive-(negative-)inequality is another special case of an LA constraint where $\forall i . a_{i} \geq 0, x_{i} \geq 0, c \geq 0\left(\forall i . a_{i} \leq 0, x_{i} \leq 0, c \leq 0\right)$. We are working on the extension to bit-vector constriants.

As in the SMT-framework, the first order logic formula is abstracted conservatively into a propositional formula and given to the SAT solver. The SAT solver extracts a set of level-zero assignments, which corresponds to a set of LA constraints. From this set, we gather difference constraints, analyze them with the Bellman-Ford algorithm described in [1] and derive reduced bounds for the variable domains if possible. Among the LA constraints left over, positive- and negative-coeffient inequalites may also yield reduced upper (lower) bounds of $x_{i}$ equal to $c / a_{i}$. The remaining LA constraints are conservatively marked as not yielding any domain reduction.

Example: Users commonly declare design inputs as int, meaning a 32-bit finite integer, causing the Boolean representation of the example in Section 2 to contain 96 bits. In applying DomRed, the equality constraint is translated into two inequalities in the usual manner. Inequalities are encoded with one bit each in the SAT solver. All these bits appear in the set of level-zero assignments. Since they all correspond to difference constraints, the Bellman-Ford algorithm yields the intervals [0,61439] for src_addr, $[0,4095]$ for payload_len and $[4096,65535]$ for dest_addr. The Boolean encoding will then require 16, 12 and 16 bits respectively, totalling 44 bits in the resulting Boolean expression (more than $2 \mathrm{X}$ reduction).

DomRed may also indicate to ValueGen that the constraints are infeasible (overconstrained situation) if the SAT solver or the LA solver detects it. This is of great value to ValueGen since it can avoid building the Boolean representations altogether.

\section{Experimental Results}

We integrated our tool DomRed into ValueGen, which, in turn, is integrated with our simulator. Our benchmark set includes both System-C and System Verilog examples. The System-C examples are smaller in size; 40 out of 68 showed improvements, the rest showed no degradation. The detailed table of results is not presented here for lack of space. The System Verilog examples consist of industrial-strength customer benchmarks. Of the 34 System Verilog examples that we experimented with, 11 showed improvement and are presented in Table1 the remaining 23 showed no degradation.

We use three parameters to measure the performance impact of applying DomRednumber of bits, CPU times and memory used. ValueGen switches between the BDD and 
Table 1. Comparison Table of without and with Bound Reduction

\begin{tabular}{|l||r|r|r|r||r|r|r||r|r|r|}
\hline \multicolumn{1}{|c||}{} & \multicolumn{3}{|c||}{ \# of bits } & \multicolumn{3}{|c||}{ CPU (sec) } & \multicolumn{3}{c|}{ MEM (Mbytes) } \\
\cline { 3 - 11 } Design & Sim. cycles & without & with & $\%$ & without & with & $\%$ & without & with & $\%$ \\
\hline \hline design1 & 5000000 & 112 & 101 & 10 & 683.0 & 549.4 & $\mathbf{2 0}$ & 40.8 & 34.2 & $\mathbf{1 6}$ \\
\hline design2 & 1000000 & 335 & 321 & 4 & 325.5 & 319.2 & $\mathbf{2}$ & 70.6 & 53.9 & $\mathbf{2 4}$ \\
\hline design3 & 50000 & 491 & 301 & 39 & 412.3 & 333.4 & $\mathbf{1 9}$ & 103.1 & 93.5 & $\mathbf{9}$ \\
\hline design4 & 1000000 & 54 & 40 & 26 & 180.9 & 174.1 & $\mathbf{4}$ & 37.2 & 37.8 & -2 \\
\hline design5 & 1000000 & 64 & 60 & 6 & 86.1 & 44.0 & $\mathbf{4 9}$ & 33.2 & 33.6 & -1 \\
\hline design6 & 1000000 & 64 & 60 & 6 & 75.9 & 48.1 & $\mathbf{3 7}$ & 33.2 & 33.7 & -1 \\
\hline design7 & 1000000 & 16 & 14 & 12 & 340.2 & 344.6 & -1 & 37.0 & 33.8 & $\mathbf{9}$ \\
\hline design8 & 44000 & 7 & 5 & 29 & 967.2 & 966.7 & 0 & 115.0 & 116.4 & -1 \\
\hline design9 & 400000 & 8484 & 8428 & 1 & 607.1 & 559.6 & $\mathbf{8}$ & 62.3 & 62.0 & 0 \\
\hline design10 & 40 & 160 & 97 & 39 & 648.5 & 603.3 & $\mathbf{7}$ & 809.1 & 756.2 & $\mathbf{7}$ \\
\hline design11 & 2500 & 374 & 335 & 10 & 234.6 & 186.3 & $\mathbf{2 1}$ & 370.7 & 282.1 & $\mathbf{2 4}$ \\
\hline
\end{tabular}

SAT solver based on the Boolean representation size to maximize the size constraints that can be solved and optimize the speed of constraint solving (better with BDDs). Our experimental results show the improvement over the default optimized algorithm. However, this makes comparing the Boolean representation sizes harder since different solvers may be used when DomRed is applied. We are working on addressing this problem to obtain a tighter comparison.

Column 1 of Table 1 specifies the design, Column 2 shows the number of simulation cycles, Columns 3-5 show the reduction of the number of bits in the constraints. Note that the number of bits is measured for the constraints only and the design may have several thousand more bits. Columns 6-8 show the CPU times and Columns 9-11 the memory reduction. The time taken by DomRed is negligibly small and hence, not presented here. The CPU time includes simulation time only in 2/11 cases, hence the CPU time improvement for most examples is for constraint solving alone.

The table shows that the reduction in the number of bits is sometimes substantial, upto $39 \%$. Smaller constraints yield better CPU times and memory reductions. Given that DomRed takes negligible time, 11/34 examples show improvement on applying DomRed and the remaining 23 examples are no worse off, we conclude that DomRed is a cheap preprocessing technique and that it is always beneficial to apply it. These results are encouraging and as part of future work, we hope to apply more powerful static analysis to reduce the size of the Boolean representation even further.

\section{References}

[1] Kim, H., Somenzi, F.: Finite instantiations for integer difference logic. In: Formal Methods in Computer Aided Design (FMCAD 2006), San Jose, CA, pp. 31-38 (November 2006)

[2] Kitchen, N., Kuelhmann, A.: Stimulus generation for constrainted random simulation. In: ICCAD (2007)

[3] Yuan, J., Shultz, K., Pixley, C., Miller, H., Aziz, A.: Modeling design constraints and biasing in simulation using bdds. In: ICCAD, pp. 584-590 (1999) 